OUTP-93-41S

\title{
Continuously Varying Exponents for Oriented Self-Avoiding Walks
}

\author{
JoHN CARDY \\ All Souls College \\ and \\ Department of Physics \\ Theoretical Physics \\ 1 Keble Road \\ Oxford OX1 3NP, UK
}

\begin{abstract}
A two-dimensional conformal field theory with a conserved $U(1)$ current $\vec{J}$, when perturbed by the operator $\vec{J}^{2}$, exhibits a line of fixed points along which the scaling dimensions of the operators with non-zero $U(1)$ charge vary continuously. This result is applied to the problem of oriented polymers (self-avoiding walks) in which the short-range repulsive interactions between two segments depend on their relative orientation. While the exponent $\nu$ describing the fractal dimension of such walks remains fixed, the exponent $\gamma$, which gives the total number $\sim N^{\gamma-1} \mu^{N}$ of such walks, is predicted to vary continuously with the energy difference.
\end{abstract}




\section{INTRODUCTION.}

Critical exponents which change continuously as a parameter of the underlying hamiltonian is varied are, by now, a well-understood phenomenon in the theory of critical behaviour. In the framework of the renormalisation group, they correspond to the existence of continuous manifolds of fixed points, rather than isolated points. In the earliest examples found, e.g. the low-temperature phase of the two-dimensional XY model, and the critical line of the Ashkin-Teller model, these manifolds are one-dimensional, but, with the development of conformal field theory (CFT), it was realised that it is possible for the moduli space of such fixed points to be multi-dimensional. The theorem of Friedan, Qiu and Shenker ${ }^{1}$ shows that, in a unitary CFT, continuously varying exponents cannot arise when the central charge $c<1$. Indeed, the examples alluded to above are now known ${ }^{2}$ to correspond to a $c=1$ theory, that of free scalar field compactified on a circle, with action

$$
S_{G}=\frac{g}{4 \pi} \int(\partial \phi)^{2} d^{2} z
$$

Unitary theories with higher dimensional moduli spaces of fixed points have larger values of $c$. While these are of great interest for string theory, their applicability to critical phenomena appears limited.

In this paper we point out that continuously varying exponents also may arise in theories with $c<1$, as long as they are non-unitary. In particular, they occur in the $O(2 n)$ model, with $-1 \leq n \leq 1$, which has a statistical interpretation as a gas of loops, and whose $n \rightarrow 0$ limit corresponds to the problem of self-avoiding walks (SAW). The main point is that a sufficient condition for a given CFT to be part of a larger moduli space of such theories is that it possess a conserved current, with complex components $(J, \bar{J})$, which generates a $U(1)$ symmetry of the theory. For, in that case, as will be shown, when a term $\lambda \int: J \bar{J}: d^{2} z$ is added to the action, the perturbation is 'truly' marginal in that its renormalization group flow vanishes to all orders, and it therefore generates a line of fixed

points. In addition, the scaling dimensions $x_{q}$ of those fields of the unperturbed theory which have non-zero $U(1)$ charge $q$ then become continuously dependent on $\lambda$, according to the formula

$$
x_{q}(\lambda)=x_{q}(0)+\frac{2 \pi \lambda q^{2}}{1-2 \pi k \lambda}
$$

where $k$ is the 'chiral anomaly', given by the coefficient of the correlation function $\langle J(z) J(0)\rangle=k / z^{2}$. 
This result is in fact a special case of a more general result of Chaudhuri and Schwartz ${ }^{3}$, who showed that, for a general current $J$, a sufficient condition that $J \bar{J}$ generate a truly marginal perturbation is that the OPE $J(z) J(0)$ should contain no simple pole term proportional to $z^{-1}$. For the special case of a $U(1)$ current, this condition is satisfied by symmetry.

For the Gaussian model (1), the current components $J$ and $\bar{J}$ are simply proportional to $\partial \phi$ and $\bar{\partial} \phi$ respectively, so that the marginal perturbation simply corresponds to changing the radius parameter $g$. One may, however, also derive the marginality explicitly by considering perturbation theory in $\lambda$. Each order is given in terms of a sum of integrals over correlation functions of the unperturbed theory. These may be regulated at short distances by a suitable explicit cut-off, and at large distances, if necessary, by considering the theory on the cylinder. While the explicit integrals become increasingly difficult to evaluate, one knows that they must resum to give the expected result. Now, in the case of a more general theory, perturbed by $J \bar{J}$, the $U(1)$ Ward identities completely fix the form of the correlation functions with an arbitrary number of insertions of this operator. Apart from a trivial relabelling of the coupling constant and the charges, these become identical with those of the $c=1$ theory. Therefore we need do no more work in evaluating them: the existence of a line of fixed points and the result (2) for the variation of the scaling dimensions follow immediately.

When applied to CFTs based on models with a symmetry group which contains $U(1)$ as a subgroup, for example $S U(N)$ WZWN models, this result is not new: is gives a part of the well-known moduli space of such theories. However, there exist also CFTs with $c<1$ which possess conserved $U(1)$ currents. The $O(2 n)$ model referred to above may be more profitably viewed as a complex $O(n)$ model. In that guise, it is equivalent to a gas of oriented loops, in which both orientations are summed over with equal weights. Examination of the partition function of this model on, for example, honeycomb lattice, shows that the loops are self-avoiding, with a fugacity $n$ for each loop, and that their interactions, in this unperturbed model, are independent of their orientation. Now consider putting a unit current around each loop, in the direction of its orientation ${ }^{4}$. This corresponds in the complex $O(n)$ model to a conserved current whose integral generates the $U(1)$ phase symmetry of the complex-valued fundamental fields. In this language, the marginal perturbation $J \bar{J}$ is the continuum limit of an short-range interaction $\lambda_{0}$ between neighboring loops which now depends on their relative orientation. In two dimensions, two segments of an oriented self-avoiding walk may approach each other in two ways: their respective ori- 
entations may either be parallel or anti-parallel (see Fig. 1). Adding such a $J \bar{J}$ term to the action corresponds to weighting these two possibilities by different amounts. The general result described above then implies that the scaling dimensions of operators with non-zero $U(1)$ charge will depend continuously on this energy difference $\lambda_{0}$. These operators will act as sources and sinks for the current, and therefore correspond to the end-points of such oriented self-avoiding walks (SAWs). Thus we predict that that the exponent $\gamma$, which relates to the total number $c_{N}$ of open SAW of length $N$ via the formula $c_{N} \sim N^{\gamma-1} \mu^{N}$, will depend continuously on the energy difference. Similarly, one may consider 'star' oriented polymers, formed by the joining of $q$ oriented SAW at their sources. The corresponding exponent $\gamma_{q}$ also depends continuously on $\lambda_{0}$, according to the law

$$
\gamma_{q}(\lambda)=\gamma_{q}(0)-2 \pi q(q+1) \lambda
$$

where $\lambda$ is a non-universal monotonic function of $\lambda_{0}$. Thus, although the various exponents are non-universal, through elimination of $\lambda$ there do exist universal relations between them, as with other examples of one-dimensional manifold of fixed points.

On the other hand, the exponent $\nu$, which determines the fractal dimension $\nu^{-1}$ of SAWs, is related to the scaling dimension of the energy operator, which has zero charge and therefore should remain constant. This is consistent with the observation that for selfavoiding loops, whose statistics are determined by correlations of energy operators, only anti-parallel contacts are possible, and they are therefore insensitive to the perturbation. (Merely changing the interaction energy for anti-parallel contacts is believed to correspond to an irrelevant perturbation, as long as it remains repulsive.)

The outline of this paper is follows. In Sec. 2 we first analyse the perturbation theory for a marginal perturbation in the $c=1$ theory. We then generalise to that of a general model with a $J \bar{J}$ perturbation, and show how the perturbative expansion is simply related to that of the case $c=1$. Making this correspondence, we deduce the relation (2). In the second part of this Section, we show how this shift in the scaling dimensions may be explained in terms of a deformation of the stress tensor, and show that nevertheless the central charge $c$ is not affected, as expected from Zamolodchikov's $c$-theorem ${ }^{5}$. While this shows how things work from the point of view of CFT, this subsection is not necessary for the subsequent development and may be omitted. In Sec. 3 we discuss how all this applies to the $O(n)$ model, and in particular the dilute limit $n \rightarrow 0$. Finally, in Sec. 3 we make some concluding remarks. 


\section{ANALYSIS OF $J \bar{J}$ PERTURBATION.}

We first discuss in detail the example of the $c=1$ Gaussian model (1). All correlation functions of this theory are related by Wick's theorem to that of the fundamental field $\langle\phi(z, \bar{z}) \phi(0)\rangle=-(1 / g)(\ln z+\ln \bar{z})$. In particular, the two-point function of the operator $V_{q} \equiv: e^{i q \phi}$ : has the form $\left\langle V_{q}(r) V_{-q}(0)\right\rangle \sim r^{-q^{2} / g}$ so that this operator has scaling dimension $x_{q}(g)=q^{2} / 2 g$. The theory possesses a $U(1)$ invariance corresponding to constant shifts in $\phi$, and the current generating this symmetry is $J_{\mu}=2 i g \partial_{\mu} \phi$. Its normalization is fixed by the operator product expansion (OPE) of $J$ with the operator $V_{q}$, which has $U(1)$ charge $q$ :

$$
J(z) \cdot V_{q}(0)=\frac{q}{z} V_{q}(0)+\cdots
$$

In this normalization, the $U(1)$ charge is $(1 / 2 \pi) \int J_{0} d x^{1}$, with the factor of $(1 / 2 \pi)$ conventional to conformal field theory.

Now set $g=g_{0}$, corresponding to the unperturbed theory, and consider the effect on the correlation function $G_{q}(r)=\left\langle V_{q}(r) V_{-q}(0)\right\rangle$ of adding a term $\delta S=\lambda \int: J \bar{J}: d^{2} z$ to $S_{G} \cdot{ }^{*}$ The result may be expressed as a perturbative expansion in $\lambda$ of the form

$$
G_{q}(r)=\sum_{n} G_{q}^{(n)}(r) \lambda^{n}
$$

where $G_{q}^{(n)}$ is given by an integral over the connected correlation function with $n$ insertions of $J \bar{J}$. For example

$$
G_{q}^{(1)}\left(z_{1}-z_{2}\right)=4 g_{0}^{2} \int\left\langle V_{q}\left(z_{1}, \bar{z}_{1}\right) V_{-q}\left(z_{2}, \bar{z}_{2}\right): \partial \phi(z) \bar{\partial} \phi(\bar{z}):\right\rangle d^{2} z
$$

Using Wick's theorem, there are four terms, corresponding to the cases where $\phi(z)$ and $\phi(\bar{z})$ are contracted onto $V_{q}$ and $V_{-q}$ respectively. After some simple algebra, these may be combined to give

$$
-q^{2} \int \frac{\left|z_{1}-z_{2}\right|^{2}}{\left|z-z_{1}\right|^{2}\left|z-z_{2}\right|^{2}} d^{2} z
$$

As it stands, this integral is in need of ultraviolet regularization, which may be implemented with a simple cut-off $\left|z-z_{i}\right|>a$. The resulting singular dependence is then of the form

$$
-4 \pi q^{2} \ln \left(\left|z_{1}-z_{2}\right| / a\right)
$$

* Normal ordering is defined in the standard way by point-splitting and subtracting the singular terms in the OPE. This depends on the coupling constant $g$, and, by convention, all normal ordering is defined in the unperturbed theory. 
The dependence on $a$ may be absorbed into an extra multiplicative renormalization of the operators $V_{ \pm q}$. The dependence on $\left|z_{1}-z_{2}\right|$ is consistent with a shift in the scaling dimension

$$
x_{q}=\frac{q^{2}}{2 g_{0}}+2 \pi \lambda q^{2}+O\left(\lambda^{2}\right)
$$

Of course, we could have obtained this another way, by simply observing that the perturbation corresponds to a shift in the coupling constant

$$
g_{0} \rightarrow g=g_{0}-4 \pi \lambda g_{0}^{2}
$$

which is consistent with (9).

The next order contribution is proportional to

$$
\int\left\langle V_{q}\left(z_{1}, \bar{z}_{1}\right) V_{-q}\left(z_{2}, \bar{z}_{2}\right): \partial \phi(z) \bar{\partial} \phi(\bar{z}):: \partial \phi\left(z^{\prime}\right) \bar{\partial} \phi\left(\bar{z}^{\prime}\right):\right\rangle_{c} d^{2} z d^{2} z^{\prime}
$$

The different kinds of Wick contraction are now illustrated in Fig. 2. Those of type (a) give a further multiplicative renormalization of $V_{ \pm q}$. Diagram (b) is clearly proportional to the square of the first order contribution, and corresponds to the next term in the exponentiation of (9). Contributions of type (c) are new, and involve the correlation function $\left\langle J(z) J\left(z^{\prime}\right)\right\rangle=k_{G} / z^{2}$, where $k_{G}=2 g_{0}$. Explicit evaluation of these integrals, in the limit $\left|z_{1}-z_{2}\right| \gg a$, is straightforward but lengthy. They give rise to a contribution proportional to $\lambda^{2} q^{2} r^{-q^{2} / g_{0}} \ln (r / a)$, where $r=\left|z_{1}-z_{2}\right|$. However, it is not necessary to perform the integrals explicitly, since we know from (10) that such terms must come from the next order in the expansion of

$$
x_{q}(g)=\frac{q^{2}}{2\left(g_{0}-4 \pi \lambda g_{0}^{2}\right)}=\frac{q^{2}}{2 g_{0}}+2 \pi \lambda q^{2}+8 \pi^{2} \lambda^{2} g_{0} q^{2}+\cdots
$$

Now consider the case of a general theory with a $U(1)$ current, perturbed by $\lambda J \bar{J}$. Let us consider again the perturbative expansion of the two-point function $\left\langle V_{q}\left(z_{1}\right) V_{-q}\left(z_{2}\right)\right\rangle$, where now $V_{q}$ represents any primary operator of charge $q$ with respect to the chosen $U(1)$ symmetry. Note that, in a given CFT, there may be more than one such operator: in that case, we choose a basis such that $\left\langle V_{q}^{i}\left(z_{1}\right) V_{-q}^{j}\left(z_{2}\right)\right\rangle \propto \delta_{i j}$. It is then straightforward to show that the perturbation does not mix these operators. Note also, that in a general theory, the unperturbed scaling dimension $x_{q}(\lambda=0)$ is not necessarily proportional to $q^{2}$. The perturbation expansion is once again given in terms of integrals over connected correlation 
functions with arbitrary numbers of insertions of $J \bar{J}$. The main point is now that these are completely determined by the $U(1)$ Ward identities and the OPE

$$
J(z) J\left(z^{\prime}\right)=\frac{k}{\left(z-z^{\prime}\right)^{2}}+O\left(\left(z-z^{\prime}\right)^{0}\right)
$$

where the $U(1)$ anomaly number $k$ is fixed by the theory. This result is most easily proved by induction. Consider the correlation function with $n$ insertions of $J$

$$
\left\langle J(z) J\left(z_{2}\right) \ldots J\left(z_{n}\right) \prod_{j} V_{q_{j}}\left(\zeta_{j}\right)\right\rangle
$$

Regarded as function of $z$, this is analytic apart from simple poles at $z=\zeta_{j}$, whose residue is given by the $\mathrm{OPE} J(z) V_{q_{j}}\left(\zeta_{j}\right)=\left(q_{j} / z-\zeta_{j}\right) V_{q_{j}}\left(\zeta_{j}\right)+\cdots$, and double poles at $z=z_{i}$, whose coefficient is given by (13). In addition, this function must behave as $z^{-2}$ as $z \rightarrow \infty$, as may be seen from its covariance under the conformal transformation $z \rightarrow z^{-1}$. Thus it is completely determined by the coefficients of its singular behaviour, and these, in turn, are determined by similar correlation functions with lower values of $n$.

A similar result applies to insertions of $\bar{J}$. For simultaneous insertions of $J$ and $\bar{J}$, we also need the contact term

$$
J(z) \bar{J}\left(\bar{z}^{\prime}\right)=(\pi k / 2) \delta^{(2)}\left(z-z^{\prime}\right)
$$

which follows from (13) and the conservation law $\bar{\partial} J+\partial \bar{J}=0$. We see then that all correlation functions with arbitrary insertions of $J$ and $\bar{J}$ are identical with those of the Gaussian model, with the sole exception that $k_{G}=2 g_{0}$ is replaced by $k$. The same is therefore true of : $J \bar{J}$ : insertions. Thus these integrals may be evaluated with no work. The scaling dimensions of operators with charge $q$ are modified. The shift may be determined by writing (12) as

$$
x_{q}(\lambda)=\frac{q^{2}}{2\left(g_{0}-4 \pi \lambda g_{0}^{2}\right)}=x_{q}(0)+\frac{2 \pi \lambda q^{2}}{1-4 \pi \lambda g_{0}}
$$

and simply making the replacement $g_{0} \rightarrow k / 2$ in the denominator of the second term. This is because, as we argued above, the higher order terms in the expansion all come from diagrams where some of the $J_{\mathrm{s}}$ are contracted onto other $J_{\mathrm{s}}$, and this involves the actual $U(1)$ anomaly $k$ of the theory, rather than $2 g_{0}$. This then gives the main result (2) quoted in the Introduction.

Perturbed stress tensor.

This result may also be seen in terms of a modification of the stress tensor $T(z)$ of the original theory. As above, we first analyse this in the $c=1$ theory. The stress tensor of 
the Gaussian model is $T(z)=-g:(\partial \phi)^{2}$.. The normalization of this may be checked by considering the OPE with $V_{q}$, for example, which gives

$$
T(z) \cdot V_{q}(0)=\frac{q^{2} / 4 g}{z^{2}} V_{q}(0)+\cdots
$$

consistent with the scaling dimension $x_{q}=q^{2} / 2 g$. As before let us write this as

$$
T=-\left(g_{0}-4 \pi \lambda g_{0}^{2}\right):(\partial \phi)^{2}: \equiv T_{0}-\pi \lambda: J^{2}:
$$

To lowest order in $\lambda$, we have the OPEs

$$
\begin{aligned}
T_{0} \cdot V_{q} & =\frac{q^{2} / 4 g_{0}}{z^{2}} V_{q}+\cdots \\
: J^{2}: \cdot V_{q} & =\frac{q^{2}}{z^{2}} V_{q}+\cdots
\end{aligned}
$$

but there are also contributions of higher order in $\lambda$ to the $O\left(z^{-2}\right)$ terms which come from insertions of $J \bar{J}$. Once again, the integrals involved rapidly become tedious to compute, but we know that they must sum to the result in (17). Therefore, to all orders in $\lambda$, (19) is replaced by

$$
\begin{gathered}
T_{0} \cdot V_{q}=\frac{q^{2} / 4 g_{0}}{\left(1-4 \pi \lambda g_{0}\right)^{2} z^{2}} V_{q}+\cdots \\
: J^{2}: \cdot V_{q}=\frac{q^{2}}{\left(1-4 \pi \lambda g_{0}\right)^{2} z^{2}} V_{q}+\cdots
\end{gathered}
$$

so that, combining these, we find

$$
T \cdot V_{q}=\left(T_{0}-\pi \lambda: J^{2}:\right) \cdot V_{q}=\frac{q^{2}}{\left(g_{0}-4 \pi \lambda g_{0}^{2}\right) z^{2}} V_{q}+\cdots
$$

consistent with the known shift in the scaling dimension.

For a more general theory perturbed by $\lambda J \bar{J}$ this result may now be taken over simply, with $\left(q^{2} / 4 g_{0}\right)$ replaced by $x_{q}(0) / 2$, and the denominator $1-4 \pi \lambda g_{0}$ replaced by $1-2 \pi \lambda k$. For example, consider the $O(\lambda)$ correction to $T_{0} \cdot V_{q}$, which involves the integral

$$
\int T_{0}(z) J\left(z_{1}\right) \bar{J}\left(z_{1}\right) V_{q}(0) d^{2} z_{1}
$$

The important contributions come from the contact term in the contraction of $T$ with $\bar{J}$ :

$$
T_{0}(z) \bar{J}\left(z_{1}\right)=(\pi / 2) \delta^{(2)}\left(z-z_{1}\right) J\left(z_{1}\right)
$$


which follows from the OPE $T \cdot J$ and current conservation. Thus this term is proportional to $q^{2}$ with a universal coefficient. However, the higher order terms in $\lambda$ all involve contractions of $J$ with $J$. and therefore depend also on $k$. The form of the full answer may be seen by writing the coefficient of $\left(1 / z^{2}\right) V_{q}$ on the right hand side of (21) as

$$
\frac{q^{2}}{4 g_{0}}+\frac{2 \pi \lambda-4 \pi^{2} \lambda^{2} g_{0}}{\left(1-4 \pi \lambda g_{0}\right)^{2}} q^{2}
$$

then replacing the first term by $x_{q}(0)$, and $g_{0}$ in the last term by $k / 2$. A similar result holds for $: J^{2}: \cdot V_{q}$. By construction, the complicated terms proportional to $q^{2}$ will then simplify in the sum $\left(T_{0}-\pi \lambda: J^{2}:\right) \cdot V_{q}$, with a result consistent with (2).

A similar calculation may be done for the central charge of the perturbed theory, defined as the coefficient $c$ of the two-point function $\langle T(z) T(0)\rangle=c /\left(2 z^{4}\right)$. According to Zamolodchikov's $c$-theorem ${ }^{5}$, this should not change along a manifold of fixed points. Writing $T=T_{0}-\pi \lambda: J^{2}$ : as before, in the Gaussian model we have

$$
\left\langle T_{0}(z) T_{0}(0)\right\rangle=\frac{g_{0}^{2}}{g^{2}} \frac{1}{2 z^{4}}=\frac{1}{\left(1-4 \pi \lambda g_{0}\right)^{2}} \frac{1}{2 z^{4}}
$$

In the general case, the $O\left(\lambda^{0}\right)$ term is of course proportional to the central charge $c_{0}$ of the unperturbed theory. However, the $O(\lambda)$ term arises from an integration over the connected part of $\left\langle T T: J^{2}:\right\rangle$. Since only the connected part is included, this does not depend on $c_{0}$, but only the (universal) scaling dimension of : $J^{2}$ :. Similarly, all the other higher order terms may involve $\langle J J\rangle$ contractions, but not $\left\langle T_{0} T_{0}\right\rangle$, and therefore are independent of $c_{0}$. We conclude that in the general case, (25) is replaced by

$$
\left\langle T_{0}(z) T_{0}(0)\right\rangle=\left[c_{0}+\left(\frac{1}{(1-2 \pi \lambda k)^{2}}-1\right)\right] \frac{1}{2 z^{4}}
$$

However, the other correlation functions $\left\langle T_{0}: J^{2}:\right\rangle$ and $\left\langle: J^{2}:: J^{2}:\right\rangle$ involve only $k$ and not $c_{0}$. As a result, when the four contributions are summed, all the $k$-dependent pieces cancel just as they do in the Gaussian theory, with the result that $c=c_{0}$.

\section{APPLICATION TO ORIENTED SAWS.}

In this section, we apply these results to the complex $O(n)$ model. On a lattice, this may be described by a partition function of the form

$$
Z=\operatorname{Tr} \prod_{r, r^{\prime}}\left(1+x s_{a}(r) s_{a}^{*}\left(r^{\prime}\right)+\text { cc. }\right)
$$


where $s_{a}(r)$ is a complex $n$-component spin at the site $r$, normalized so that $\operatorname{Tr} s_{a}(r) s_{b}^{*}\left(r^{\prime}\right)=$ $\delta_{a b} \delta_{r r^{\prime}}$, and the product is over nearest neighbour pairs $\left(r, r^{\prime}\right)$. When expanded in powers of $x, Z$ may be expressed as a sum over configurations of oriented self-avoiding loops, with each link weighted by a factor $x$ and each loops by a factor $n$. In the limit $n \rightarrow 0$ we have the problem of a single self-avoiding loop. This theory has an obvious $U(1)$ symmetry under phase rotations $s_{a} \rightarrow e^{i \alpha} s_{a}$, and the corresponding current is the lattice version of $J_{\mu}=(i / 2)\left(s_{a}^{*} \partial_{\mu} s_{a}-s_{a} \partial_{\mu} s_{a}^{*}\right)$. It corresponds to a unit current along each loop in the direction of its orientation.

In addition to closed loops, one may also consider open oriented SAWs. A source at which $q$ distinct SAWs originate is related in the $O(n)$ model to the insertion of an operator $\phi_{a_{1} \ldots a_{q}}^{(q)} \equiv s_{a_{1}} \ldots s_{a_{q}}$, which has charge $q$ under this $U(1)$ symmetry. More precisely, the correlation function

$$
\left\langle\phi^{(q)}(0) s_{a_{1}}^{*}\left(r_{1}\right) \ldots s_{a_{q}}^{*}\left(r_{q}\right)\right\rangle
$$

gives the generating function for the number of 'star' oriented polymers which originate at $r=0$ and have their ends at $r_{1}, \ldots, r_{q}$. In the absence of any orientation-dependent interactions, the sum over both orientations of each loop is equivalent to a problem of unoriented loops, and the complex $O(n)$ model is equivalent to a real $O(2 n)$ model. In that case, the critical limit of this model is known to be described in the continuum limit by a conformal field theory with $O(2 n)$ symmetry, which may be derived by making an appropriate modification of the Gaussian model, or Coulomb gas, with a charge on the boundary. From this analysis, the scaling dimensions of the operators $\phi^{(q)}$ are known to $\mathrm{be}^{6}$

$$
x_{q}(0)=\frac{g q^{2}}{8}-\frac{(g-1)^{2}}{2 g}
$$

where $n=-\cos \pi g$ with $1 \leq g \leq 2$. This gives the exponent $\gamma_{q}=\nu\left(2-x_{q}(0)-q x_{1}(0)\right.$ which appears in the asymptotic behaviour of the number $c_{N}^{(q)} \sim N^{\gamma_{q}-1} \mu^{N}$ of such star polymers. Of course, $q=1$ corresponds to the usual case of a linear polymer, with $\gamma_{1}=\gamma=\nu(2-\eta)$.

Now consider adding an interaction energy between different parts of loops which depends on their relative orientation. In general, this may be written as

$$
\sum_{r, r^{\prime}} F\left(r-r^{\prime}\right) J_{\mu}(r) J_{\mu}\left(r^{\prime}\right)
$$

where the function $F$ is assumed to be short-ranged. In describing the continuum limit of the effects of this perturbation, we may use the OPE

$$
J_{\mu}(r) J_{\mu}\left(r^{\prime}\right)=\text { singular term }+: J \bar{J}:+\cdots
$$


The singular term leads to a trivial constant in the energy, and may be subtracted off. The terms represented by dots are all irrelevant. We are therefore left with precisely the type of perturbation described in the preceding section, with a bare coupling constant $\lambda_{0}=\sum_{r} F(r)$. It should be stressed that this is not necessarily equal to the renormalized coupling constant $\lambda$ introduced in Sec. 2 , since it will be renormalised in a non-universal way by the irrelevant couplings. We do expect $\lambda_{0}$ and $\lambda$ to have the same sign, however.

The introduction of $\lambda_{0}$ will also, in general, give additional mass renormalisation, and hence will shift the value of the bare mass at which the renormalised mass vanishes. This corresponds to a shift in the critical value $x_{c}$ of the fugacity $x$ of the lattice model, and hence of the connective constant $\mu=x_{c}^{-1}$. We may avoid this problem by considering the following special case. Consider SAW on the square lattice. In this model, the walks are never allowed to traverse the same edge more than once. However, it is possible to have configurations where opposite edges of an elementary square are occupied by different parts of the same walk (or, for $n \neq 0$, by parts of different walks.) Call this a close encounter. When this happens, the opposite edges may be traversed either in a parallel, or an anti-parallel, fashion (see Fig. 1.) In the ordinary SAW problem, each of these configurations is assigned unit weight. Now consider giving each parallel close encounter a weight $w=e^{-\lambda_{0}} \neq 1$, meanwhile continuing to assign anti-parallel encounters unit weight. This will then correspond to an energy difference of the kind we require, as well as an overall shift in the net repulsive energy (an irrelevant perturbation.) It may be shown that these two effects will cancel in their contribution to mass renormalization, at least for $w<1$, and hence the connective constant $\mu$ will be independent of $w$. This will certainly be true for closed loops, which can contain no parallel close approaches and are therefore insensitive to $w$. For single open walks, let us define $c_{N, M}$ to be the number of such walks with $N$ steps and $M$ such parallel close encounters. Thus, we are interested in the asymptotic behaviour of $c_{N}(w)=\sum_{M} c_{N, M} w^{M}$. The following argument is due to A. Owczarek ${ }^{7}$ Clearly $c_{N, 0}<c_{N}(w)<c_{N}(1)$ for $0<w<1$. Both of these bounds increase as $\mu^{N}$ (apart from powers of $N$.) This is true of $c_{N}(1)$ by definition. The lower bound is itself bounded below by the number of walks which return to the vicinity of the origin This is essentially the number of closed loops of length $N$, which are known to have the same connective constant $\mu$. A similar argument should also hold for star polymers, at least for $w<1$. The attractive case $w>1$ is less clear. For sufficiently large $w$ we expect that bound states can form between two or more parallel walks. In that case, the critical point will occur when the bound state mass vanishes rather than that of a single particle, and this may occur at a smaller value of $x_{c}$. Certainly, for sufficiently large $w$, 
much greater than $\mu$, the dominant configurations of single walks will be those wrapped up as tightly as possible to maximise the number of parallel close encounters. In that case $c_{N}(w)$ will behave roughly as $w^{N}$, and the walks will be compact.

From the above discussion, we see that, at least for $w<1$, and perhaps for a small range of $w>1$, we may apply the results of Sec. 2 to show that the number of star polymers, weighted by $w$, behaves asymptotically as

$$
c_{N}^{(q)}(w) \sim N^{\gamma_{q}(w)-1} \mu^{N}
$$

where $\gamma_{q}(w)=\nu\left(2-x_{q}(\lambda)-q x_{1}(\lambda)\right)$, and $x_{q}(\lambda)$ is given by formula $(2)$. Note that the energy operator $s_{a}^{*} s_{a}$ has $q=0$ and is therefore insensitive to $\lambda$. Thus the fractal dimension of these walks is the same as the ordinary case, with $\nu=\frac{3}{4}$.

For the complex $O(n)$ model the $U(1)$ anomaly number $k(n)$ has been calculated by Coulomb gas methods to beRef. 8

$$
k(n)=\frac{2 \pi(g-1) \cot \pi g}{g}=\frac{2 \pi n \arccos n}{\sqrt{1-n^{2}}(\pi+\arccos n)}
$$

where $0 \leq \arccos n \leq \pi$. (The normalization of $k$ differs from that used in Ref. 8 by a factor $\pi^{2}$.) Note that for $n=0, k$ vanishes, so that (2) simplifies to

$$
x_{q}(\lambda)=\left(\frac{9}{48}+2 \pi \lambda\right) q^{2}-\frac{1}{12}
$$

This then leads to (3) of the Introduction. For $\lambda>0$, corresponding to the repulsive case $w<1$, the value of $x_{q}$ increases and therefore $\gamma_{q}$ decreases as expected. It is interesting to note that, for the opposite case $\lambda<0$, there appears to be a value of $\lambda$ for which $x_{1}$ vanishes. It is tempting to suppose that this corresponds to the collapse transition where the single walk winds up on itself, but there is no real evidence for this. Also, because of the renormalization effects, we do not know whether this critical value of $\lambda$ occurs for a finite value of the bare coupling $\lambda_{0}$. In fact this change of sign of the scaling dimensions for sufficiently large $\lambda$ may be shown to occur for all values of $n \leq 1$.

An interesting result follows if we differentiate (32) with respect to $w$ and set $w=1$. This gives an estimate for the average number of of parallel close encounters of an oriented star polymer:

$$
\bar{M}_{N}=\frac{\sum_{M} M c_{N, M}}{\sum_{M} c_{N, M}} \sim A q^{2} \ln N
$$

where $A$ is non-universal, but independent of $q$. Since the mean number of both types of encounter increases linearly with $N$, this suggests that these effects might be rather hard to see in enumerations with small values of $N$. 


\section{CONCLUSIONS.}

In this paper we have pointed out that the existence of a non-trivial moduli space, or continuum of fixed points, is not restricted to conformal field theories with $c \geq 1$, but already occurs in non-unitary $c<1$ theories of interest in statistical mechanics. All that is required is that the theory possess a conserved $U(1)$ current. Since all values of $c>0$ may be reached by composing $c<1$ theories, it follows that continuously varying exponents may occur for all positive values of the central charge.

We have pointed out a specific application to oriented SAWs with orientation-dependent interactions. While our conclusions apply mainly to the case where the forces are repulsive, these walks appear also to undergo a new kind of collapse transition if the forces between parallel segments of the walk are sufficiently attractive. This is not the usual theta-point because it does not occur for closed loops. A similar kind of collapse transition has recently been observed in an exactly solvable lattice model of walks coupled to an Ising degree of freedom ${ }^{9}$. Note that the occurrence of what we have termed parallel close encounters in a SAW implies that at least one end is 'trapped' by the walk. Thus the number of such trapped walks is thus given by $c_{N}(1)-c_{N}(0)$, and our results indicate that a fraction $1-O\left(N^{-\gamma(0)+\gamma(1)}\right)$ are trapped. Note that this exponent is not expected to be universal since it depends on the precise definition of trapping. While the occurrence of continuously varying exponents in the context of oriented polymers is interesting, it should be noted that this effect is peculiar to two dimensions. In $d>2$ dimensions, the $\vec{J}^{2}$ interaction has dimension (length) ${ }^{2-2 d}$, and so is irrelevant.

The author acknowledges useful correspondence with A. J. Guttmann. This work was supported in part by a grant from the SERC.

\section{FIGURE CAPTIONS.}

1. An oriented self-avoiding walk. $a$ indicates a parallel close encounter; $b$ indicates an anti-parallel one. Note that only the latter are allowed for close self-avoiding loops. In the model under consideration, these two types of close encounter are assigned different weights.

2. Some of the contributions to the $O\left(\lambda^{2}\right)$ correction to the two-point function $\left\langle V_{q} V_{-q}\right\rangle$. In these diagrams, the dashed line represents the unperturbed correlation function, 
solid lines represent OPE contractions of the current with $V_{ \pm q}$, and the dotted line is a current-current contraction, proportional to $k$.

\section{REFERENCES}

1. D. Friedan, Z. Qiu and S. Shenker, Phys. Rev. Lett. 52, 1575 (1984).

2. B. Nienhuis, in Phase Transitions and Critical Phenomena, v. 11, C. Domb and J. L. Lebowitz, eds. (Academic, 1986.)

3. S. Chaudhuri and J. A. Schwartz, Phys. Lett. B 219, 291 (1989).

4. J. Miller, J. Stat. Phys. 63, 89 (1991).

5. A. B. Zamolodchikov, Zh. Eksp. Teor. Fiz. 43, 565 (1986); [ JETP Lett. 43, 730 (1986).]

6. H. Saleur, J. Phys. A19, L807 (1986).

7. A. J. Guttmann, private communication.

8. J. L. Cardy, Oxford preprint OUTP-93-34S.

9. B. Nienhuis, private communication. 


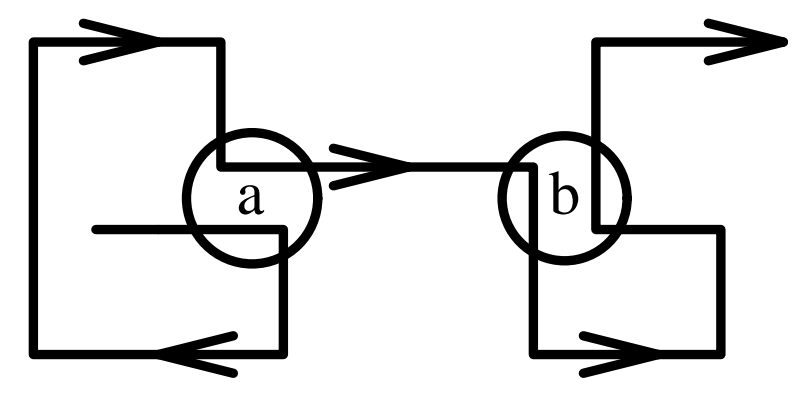

Figure 1 


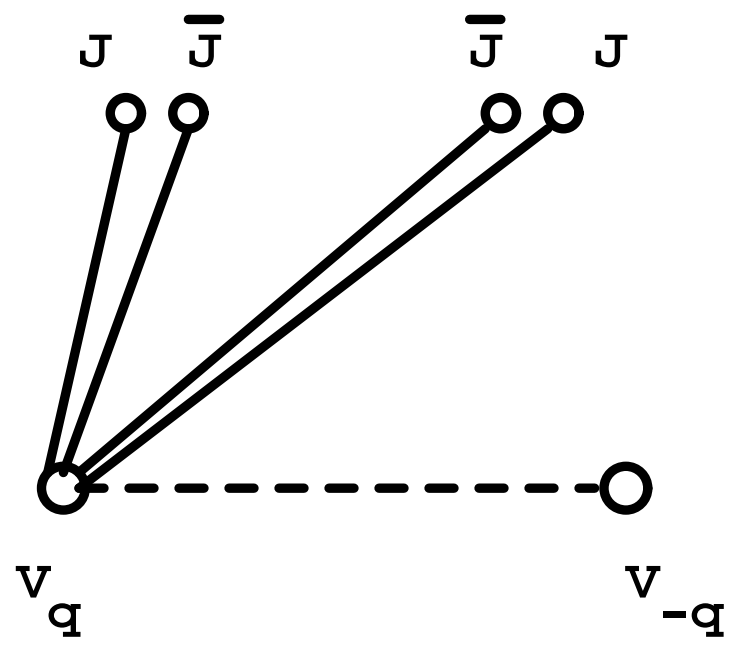

(a)

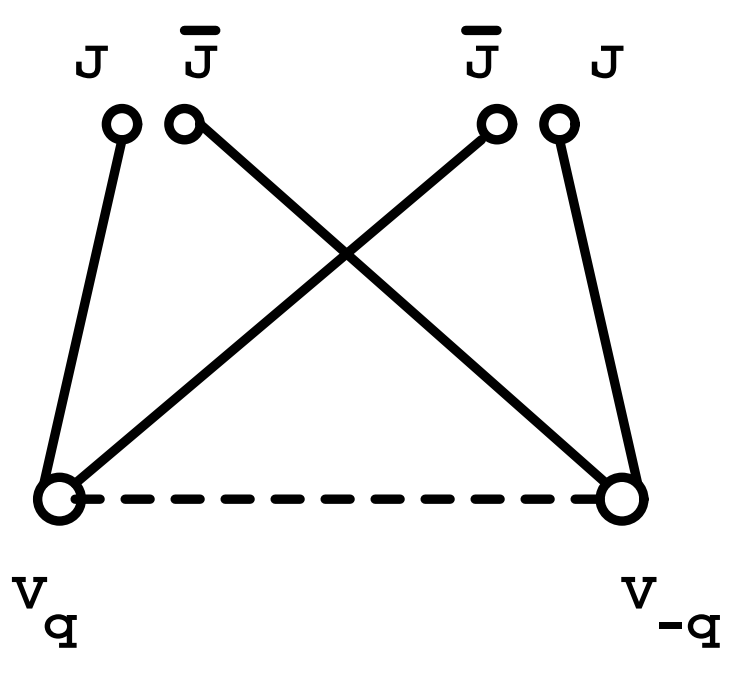

(b)

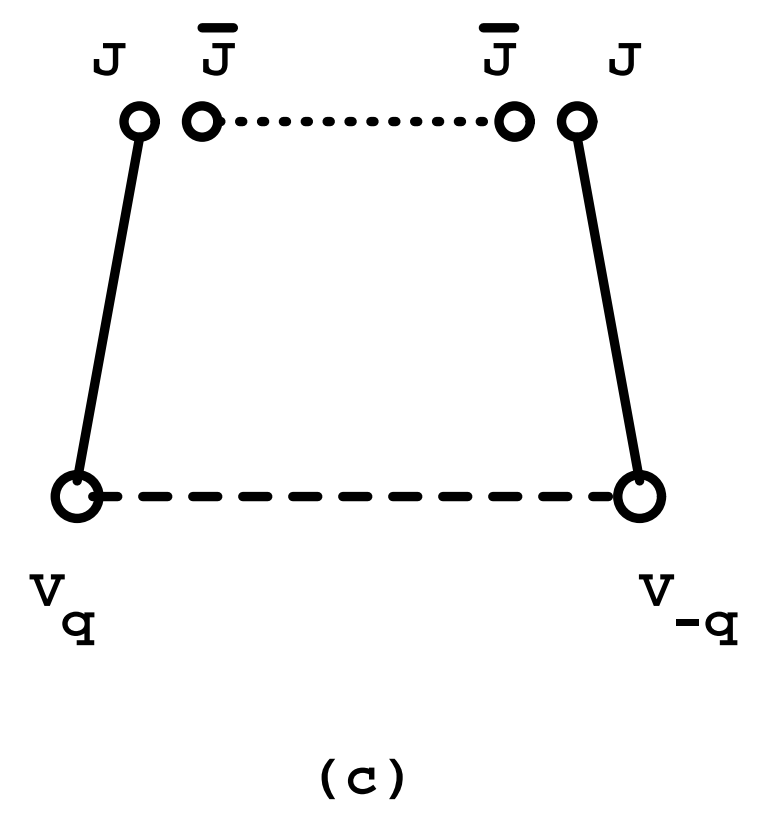

Figure 2 